\title{
EVALUACIÓN DE LA PROPUESTA DE ENSEÑANZA DE LAS ÁREAS DE LENGUAJE Y MATEMÁTICAS EN LA INSTITUCIÓN EDUCATIVA SAN JOSÉ DEL PANTANO
}

\author{
Evaluation of the Language and Math Teaching Proposal at San José del Pantano Education \\ Institution
}

Avaliação da proposta de ensino das áreas de linguagem e matemáticas na Instituição Educativa San José del Pantano

RECIBIDO:29 DEJULIO 2015

es

\section{RESUMEN}

El propósito de este artículo es presentar los avances de un estudio tendiente a evaluar el impacto del programa Todos a Aprender en los procesos de enseñanza del lenguaje y las matemáticas en los docentes de básica primaria de la Institución Educativa San José del Pantano del municipio de Puerto Escondido, en el departamento de Córdoba. Esta investigación tiene un enfoque cualitativo, con un diseño de estudio de caso, con tres categorías de análisis: comunidad de aprendizaje de docentes, metodología del estudio de clases y desarrollo profesional situado. Para su desarrollo, se tienen en cuenta las siguientes fases: proyección de las comunidades de aprendizaje, ejecución del desarrollo profesional situado y consolidación de la comunidad de aprendizaje de docentes. Se muestran los resultados obtenidos acerca la formación docente y el análisis comparativo de las pruebas saber de los grados $3^{\circ}$ y $5^{\circ}$ durante 2014.

PALABRAS CLAVE: lenguaje, matemáticas, comunidad de aprendizaje, estudio de clases, formación docente, estudio de caso.

\section{EVALUADO:14DE SEPTIEMBRE 2015}

Alex Mauricio Díaz (Colombia)

Universidad de Córdoba

Máster en Educación

alexmau995@gmail.com

\section{en}

\section{ABSTRACT}

This article presents the progress of a study that evaluates the impact of the program Todos a Aprender (Everybody Learns) within language and math teaching processes for the elementary school teachers that belong to the San José del Pantano Education Institution in Puerto Escondido, department of Córdoba. This is a qualitative investigation, with a case study design and three analysis categories: Learning community of teachers, methodology of class study, and located professional development. We considered the following phases within its development: projection of learning communities: execution of the located professional development and consolidation of the teacher learning community. We show the results obtained regarding teacher training and the comparative analysis of the knowledge tests belonging to grades 3 and 5 during 2014.

KEYWORDS: Language, math, learning community, class study, teacher training, case study.
ACEPTADO:29 DE SEPTIEMBRE 2015

\section{por}

\section{RESUMO}

O propósito deste artigo é apresentar os avanços de um estudo tendente a avaliar o impacto do programa Todos a Aprender nos processos de ensino da linguagem e as matemáticas nos docentes de básica primária da Instituição Educativa San José del Pantano do município de Puerto Escondido, no departamento de Córdoba. Esta pesquisa tem uma focagem qualitativa, com um desenho de estudo de caso, com três categorias de análise: comunidade de aprendizado de docentes, metodologia do estudo de aulas e desenvolvimento profissional situado. Para o seu desenvolvimento, se têm em conta as seguintes fases: projeção das comunidades de aprendizado, execução do desenvolvimento profissional situado e consolidação da comunidade de aprendizado de docentes. Mostram-se os resultados obtidos acerca a formação docente e a análise comparativa das Pruebas Saber das séries $3^{\circ}$ e $5^{\circ}$ durante 2014.

PALAVRAS CHAVE: linguagem, matemáticas, comunidade de aprendizado, estudo de aulas, formação docente, estudo de caso.

\section{PARA CITAR ESTE ARTÍCULO/TO CITE THIS ARTICLE/PARA CITAR ESTE ARTIGO:}

Díaz, A. M. (2015). Evaluación de la propuesta de enseñanza en lenguaje y matemáticas en la Institución Educativa San José del Pantano. Panorama, 9(16) pp. $25-39$. 


\section{INTRODUCCIÓN}

Las metas de las políticas del Gobierno actual son alcanzar para 2025 la calidad educativa, que puede ser concretada con prácticas de aula pertinentes, eficaces y significativas, ya sea en salones de clase, ya sea dondequiera que se desarrolle una acción pedagógica sistemática dirigida a un grupo de estudiantes. El sistema educativo colombiano debe garantizar aprendizajes básicos, universalmente definidos, como las áreas del lenguaje y las matemáticas, las cuales son el sustento al considerarse transversales y esenciales para los demás aprendizajes. Estas dos áreas son los pilares de la educación básica primaria, secundaria, media y superior. Frente a esto se plantea que una de las estrategias que mejoran las prácticas de aula y el proceso de enseñanza de los docentes es la implementación de comunidades de aprendizaje y de prácticas educativas basadas en la instrucción. Por ello, debe dirigirse a los maestros un proceso de formación contextualizada y en relación con las vivencias del aula y sus problemáticas específicas.

El presente estudio se origina como una necesidad de reflexión sobre el impacto de la comunidad de aprendizaje de docentes en las prácticas de enseñanza del lenguaje y las matemáticas en básica primaria de la Institución Educativa San José del Pantano, a causa de los bajos resultados obtenidos por los estudiantes en las evaluaciones de pruebas nacionales, como Saber-Icfes grados $3^{\circ}$ y $5^{\circ}$.

\section{MARCO TEÓRICO}

Trabajar en comunidades de aprendizaje los aspectos de conceptualización, retroalimentación y estrategias de evaluación en el desarrollo de las clases dará cuenta del mejoramiento de los resultados para las pruebas Saber $3^{\circ}$ y $5^{\circ}$ que se realizan en la Institución Educativa cada año. Las comunidades de aprendizaje, compuestas principalmente por docentes de primaria, constituyen una puesta en marcha de la formación situada en los ejes de didáctica del lenguaje y matemáticas en los establecimientos educativos focalizados por el programa Todos a Aprender. Se pretende que los docentes reflexionen y discutan sobre su práctica pedagógica desde tres momentos, amparados en la metodología de estudio de clase, que son los siguientes: planeación, observación y reflexión o discusión.
Una comunidad de aprendizaje es "un proyecto de transformación social y cultural de un centro educativo y de su entorno, para conseguir una sociedad de la información para todas las personas"(Valls, 2005). Visto esto, es muy importante decir que para el desarrollo de una comunidad de aprendizaje eficaz y significativa se necesita establecer un esquema de los procesos de enseñanza y aprendizaje, para lo cual se establecen las siguientes fases: sensibilización, toma de decisiones y proyección de la comunidad de aprendizaje de los docentes de básica primaria de la Institución Educativa, selección de prioridades, planificación, investigación, formación y ejecución del desarrollo profesional a través de las sesiones de trabajo situado y consolidación de la comunidad de aprendizaje de docentes. Desde este marco, en el caso específico de la Institución Educativa San José del Pantano, se tiene que para la primera fase de proyección de las comunidades de aprendizaje de docentes, desde inicios de 2013, se han hecho socializaciones con la comunidad educativa en general, informándoles acerca de la pertinencia de la conformación de unas comunidades de aprendizaje de docentes en la Institución Educativa.

La fase de selección de prioridades, planificación, investigación, formación y ejecución del desarrollo profesional situado se ha realizado dentro de las políticas del Gobierno actual, las cuales apuntan al mejoramiento de la calidad educativa a través del uso de estrategias metodológicas actualizadas y equipos de estudio y planeación de secuencias didácticas en comunidades de aprendizaje, que atiendan a los problemas identificados en el aula mediante la implementación de la metodología de estudio de clase, en la cual se realiza el proceso de planeación, observación, ejecución y evaluación de los procesos de enseñanza de los docentes. Por último, la proyección de estas comunidades de aprendizaje es la de ser sostenible a lo largo del tiempo y alcanzar los objetivos de mejorar los niveles de desempeños y competencias en las áreas de lenguaje y matemáticas en los estudiantes de educación básica primaria de la Institución Educativa, así como la formación docente y la actualización pedagógica, garantizando de este modo la consolidación de la comunidad de aprendizaje de docentes como medio eficaz para el logro de la calidad educativa en el plantel.

El desarrollo de este estudio busca evaluar la formación de los docentes en cuanto a las nuevas estrategias pedagógicas y el uso de los materiales educativos y las herramientas didácticas para el mejoramiento del desarrollo 
de sus clases, lo cual conlleva también mejorar el desempeño de los estudiantes en sus niveles de aprendizaje de las áreas del lenguaje y las matemáticas. La valoración del trabajo investigativo se definirá por la comunidades de aprendizaje de docentes en la Institución Educativa y su impacto en estudiantes, docentes y padres de familia de la comunidad estudiantil, estableciendo si se pudo alcanzar la transformación de los procesos de enseñanza y el mejoramiento de la calidad educativa de los estudiantes en las áreas del lenguaje y las matemáticas. Para ello se parte de la siguiente pregunta: ¿cuál es el impacto del programa Todos a Aprender en los procesos de enseñanza del lenguaje y las matemáticas en la Institución Educativa San José del Pantano”?

Como consecuencia de los bajos resultados en las Pruebas Saber-Icfes en los grados $3^{\circ}$ y $5^{\circ}$, el Ministerio de Educación Nacional (MEN) diseñó e implementó el programa Todos a Aprender, mediante el cual se ha buscado transformar y mejorar la calidad educativa de los establecimientos educativos en el país. Este programa resalta el trabajo situado con los docentes en los ejes de didáctica del lenguaje y las matemáticas en básica primaria, considerando que una sólida preparación de los estudiantes en estas dos áreas constituye la base para la transformación de la calidad educativa en la educación media y superior. A partir de los resultados obtenidos, Todos a Aprender toma como base de acción las comunidades de aprendizaje, las cuales se configuran a partir de equipos de trabajo de docentes, orientados por un docente tutor capacitado previamente por el MEN. La importancia que Todos a Aprender y las comunidades de aprendizaje tienen en las escuelas y los intentos del MEN de mejorar el sistema educativo en el país se consideran de vital importancia para llevar a cabo la presente investigación, cuyo objetivo es analizar el impacto de la comunidad de aprendizaje en la práctica de enseñanza del lenguaje y las matemáticas de los docentes de primaria en la Institución Educativa San José del Pantano.

Las comunidades de aprendizaje de docentes son claves en el fortalecimiento de los sistemas educativos. Detrás del docente en el aula se encuentra el sistema educativo anidado en una comunidad que lo fortalece. En Canadá y Australia, el concepto de comunidad de aprendizaje ha sido implementado desde la educación básica primaria hasta la cúspide de la educación superior (Calhoun, 1994; Fullan, 2005; Garmston y Wellman, 1999), de tal forma que se pueda fomentar el aprendizaje para la vida, que involucre las comunidades de aprendizaje de docentes en los diferentes niveles de la educación: primaria, básica, media y superior, con un alto porcentaje de éxito y calidad (Mitchell y Coral, 2001). El efecto democratizador de una comunidad de aprendizaje da lugar a que los procesos de aprendizaje ocurran en ambientes diversos e incluyentes para atender las visiones y necesidades de diferentes grupos étnicos y culturales.

Por otro lado, el aspecto prominente y crucial en las comunidades es el aprendizaje de los maestros. La literatura destaca que las comunidades de aprendizaje de docentes son efectivas en el desarrollo profesional del docente y liderazgo escolar (Hu-pei, 2002; CochranSmith y Lytle, 1999; Fullan, 2005). La comunidades de aprendizaje de docentes provee un ambiente fértil para desarrollar actividades de aprendizaje colaborativo entre docentes, que conllevan desarrollar procesos de investigación y acción, así como procesos de autoevaluación. Gran parte de los resultados conducen a mejoras en las prácticas de enseñanza y de evaluación formativa en el aula, que pueden mejorar el rendimiento académico estudiantil (Roberts, Pruitt, Du Four y Eaker, 1998).

En este marco, las investigaciones sobre las comunidades de aprendizaje de docentes como objeto de investigación científica iniciaron a finales de la década de 1980 en Japón, los Estados Unidos y España. La mayoría de las investigaciones se han orientado hacia el estudio de la práctica de la enseñanza de los docentes a través del aprendizaje colaborativo y del impacto de las comunidades de aprendizaje de docentes en la escuela (McKinsey, 2007; Valls, 2005). En el contexto colombiano, este temática comenzó a ser abordada y estudiada apenas hace doce años mediante el convenio del MEN y la Agencia Japonesa de Cooperación Internacional (JICA) desde 2003 para el mejoramiento del sistema de enseñanza de docentes de matemáticas y ciencias naturales.

La metodología del estudio de clase es una actividad permanente de muchos actores del sistema educacional japonés, que incluye a todos sus profesores de escuelas y colegios, a quienes permiten no solo compartir su conocimiento y aprender unos de otros, sino también aportar como investigadores al desarrollo de la educación de su país. El estudio de clase se entiende como "la investigación que tiene por objeto la clase" (Sistema de
| Evaluación de la propuesta

de enseñanza de las áreas de lenguaje $y$ matemáticas en la

Institución Educativa San José del Pantano

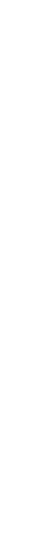


Alex Mauricio

Díaz I

Panorama I pp. 25-39 I

Volumen 9 I

Número 17|
Capacitación docente en Japón, s. f.). Es una metodología de cualificación docente que permite reflexionar sobre las prácticas de aula a partir de la planeación, la observación y el análisis de las clases. Una vez el equipo culmina el estudio de una clase, inicia un nuevo estudio que puede retomar la experiencia anterior, para mejorar y perfeccionar las clases, o puede generar un nuevo ciclo a partir de otro foco que resulte de interés para el equipo estudio de clase que complemente el problema o necesidad identificada. Se concibe como una estrategia de trabajo de un equipo que decide aprender de sus propias prácticas en beneficio de los procesos de enseñanza y aprendizaje, acordes con el fortalecimiento de las competencias de los estudiantes (MEN, 2012).

Desde los siguientes antecedentes, se busca demostrar el rastreo bibliográfico realizado en varias investigaciones realizadas acerca de las comunidades de aprendizaje y el trabajo en equipo de docentes en diferentes contextos y niveles educativos, las cuales se convertirán en sustento para determinar el estado actual del presente estudio. En primer lugar, las comunidades de aprendizaje son grupos que trabajan en torno a un propósito común; en este caso, mejorar el aprendizaje, el rendimiento académico de los estudiantes y la instrucción del docente (Nunan, 2003). Una comunidad de aprendizaje es autodidacta y crece en conocimiento y capacidad continuamente, para tener mejores herramientas que se puedan utilizar en su plan de acción. Puede estar conformada por los docentes de cualquier nivel de educación: primaria, básica y media de las instituciones educativas del sector oficial o privado y urbano o rural. Estas promueven la equidad y pertinencia en los procesos educativos con participación de la comunidad en general. También fomentan la contextualización de la educación a las realidades socioculturales de cada comunidad. De aquí que se utilicen, principalmente, como técnicas de recolección de información y de trabajo, las encuestas, las entrevistas, los cuestionarios y la observación directa.

En el ámbito latinoamericano, en Chile se encuentra la Campaña de lectura, escritura y matemática. Estrategia LEM, que es liderada por el Ministerio de Educación de ese país desde 2002, donde se tiene como objetivo general el aprendizaje colaborativo y la reflexión de la práctica educativa, así como el mejoramiento de los aprendizajes de los estudiantes en las áreas de lenguaje y matemáticas, desde preescolar hasta la educación básica (9 años de edad), por medio de la cualificación de las competencias y los conocimientos didácticos de los docentes de dichas áreas, las cuales fueron escogidas dada su relevancia en la comprensión de las otras áreas del saber y las habilidades que implican su aprendizaje para analizar, razonar y participar en los diferentes aspectos de la sociedad (Vezub, 2009). Esta política educativa pretende mejorar el rendimiento académico de los estudiantes, así como los índices de deserción y repitencia, fundamentada en tres aspectos clave: la elaboración de unidades didácticas de lenguaje y matemática con estrategias pedagógicas diseñadas por expertos, el desarrollo profesional de los docentes y el acompañamiento del trabajo didáctico en las aulas de clase.

La estrategia LEM desarrolló una metodología de trabajo situado, para lo cual se focalizaron primeramente las escuelas con bajos resultados académicos en las pruebas nacionales del Sistema de Medición de la Calidad de la Educación (Simce) y la población estudiantil de bajo nivel socioeconómico, siendo su participación en el programa de suma obligatoriedad. Así es como los docentes desde el último año de párvulos hasta $4^{\circ}$ año de primaria debían cumplir con $80 \%$ de la asistencia a las jornadas académicas programadas, cuya capacitación se les certificaba al finalizar los dos años de gestión. La intervención en las escuela se realizó en un periodo de cuatro años, de los cuales dos hacían hincapié en el trabajo curricular y la formación de los docentes en el área de lenguaje y dos en matemática, empleando para ello material pedagógico y las unidades didácticas diseñadas por las universidades aliadas (Chile, 2001).

Para el alcance de sus objetivos, la estrategia LEM se fundamenta en la metodología del apoyo profesional y el aprendizaje entre pares en el interior de las escuelas, así como en el desarrollo de talleres de formaciones en lenguaje y matemáticas a los docentes de aula. Asimismo, los directores de las escuelas son asesorados para que desde su gestión escolar se propicien las condiciones, a fin de implementar las diversas estrategias didácticas que genera este programa. Un actor primordial en este sistema es el profesor consultor, quien es un docente de aula, un par, seleccionado por su buena trayectoria laboral y perfil profesional para asumir el papel de asesor y capacitador en las escuelas de su localidad.

Este proyecto educativo se fundamenta en la concepción de que las transformaciones en el contexto pedagógico se deben dar en el quehacer didáctico, en la práctica, y 
para ello es importante contar con elementos mediadores, como las unidades didácticas, cuyo fin es servir de apoyo práctico para la cualificación de las clases con procesos definidos y materiales y contenidos definidos que sirvan como referentes y que, a partir de situaciones de enseñanza reales, conlleven modificar las prácticas. Esto implica que en el año escolar se implementen cuatro unidades didácticas, en las que cada unidad cubre entre una y dos semanas de clases y se desarrolla un taller de una de las unidades que se toma como referencia para el trabajo que los docentes van a realizar con asesoría de los profesores consultores.

Dentro de la estrategia LEM liderada por el Ministerio de Educación chileno se han alcanzado los siguientes resultados (Vezub, 2009): formación de los profesores consultores, talleres de estudio institucionales en torno a la discusión y análisis de la unidad didáctica diseñada por especialistas, talleres interinstitucionales que reúnen a tres escuelas de la zona, trabajo in situ entre el profesor del aula y el profesor consultor, talleres de retroalimentación o devolución al finalizar el desarrollo de cada unidad didáctica.

\section{LAS COMUNIDADES DE APRENDIZAJE DE DOCENTES EN COLOMBIA}

En el contexto colombiano, el estudio sobre las comunidades de aprendizaje se ha desarrollado desde un ámbito institucional a través del MEN. Para el MEN (2011), los aprendizajes efectivos de los docentes suceden en su propia práctica en el aula, por lo que las estrategias de formación situada se dan en torno a las prácticas de aula o en talleres a los cuales los docentes llevan sus problemáticas de aula para trabajarlas en comunidades de aprendizaje. En consecuencia, asumir una estructura de formación situada requiere la creación de un ambiente de intercambio y perfeccionamiento de conocimientos, actitudes y buenas prácticas en cada colectivo de maestros para alcanzar el objetivo de formar, con apoyo técnico apropiado, comunidades de aprendizaje comprometidas con procesos de mejoramiento. Entonces, la creación de una comunidad de aprendizaje de docentes permite garantizar que sea superada la barrera del frecuente aislamiento del maestro; el trabajo entre pares es metodología probada y aplicada continuamente con los estudiantes en el desarrollo de las clases dentro y fuera del aula escolar (McKinsey, 2007, citado en MEN, 2011).
Las comunidades de aprendizaje de docentes permiten que las transformaciones se sostengan en el tiempo a partir de innovaciones permanentes sobre los procesos de enseñanza. Los resultados obtenidos en la investigación realizada por el MEN en el programa Todos a Aprender concluyeron que

Las comunidades de aprendizaje son comunidades de conocimiento y de práctica; reflexionan y encuentran soluciones a las problemáticas específicas de aula en torno a los procesos de aprendizaje de los estudiantes, comparten inquietudes e identifican colectivamente alternativas pedagógicas. Algunas características de las comunidades de aprendizaje es que investigan, documentan sus experiencias, comparten sus prácticas y se nutren de las problemáticas del contexto escolar (MEN, 2011, p. 18).

Por otro lado, la implementación de la metodología del estudio de clases se ha desarrollado en diferentes regiones del país, la cual inició con la experiencia Maestro, invitame a tu clase, en el Grupo de Estudio de Clase de la Institución Educativa E1 Hatillo, y desarrollado en la básica primaria. Proceso orientado por la docente Luz Adriana Cadavid, quien asistió al Sistema de Capacitación de Profesores de Ciencias Naturales y Matemáticas, realizado en la Universidad Pedagógica de Miyagi, en Japón, en 2006. El objetivo o propósito de la implementación de esta experiencia fue el de mejorar la enseñanza de las matemáticas en los niveles de la educación básica primaria a partir de la conformación de una comunidades de aprendizaje, mediante la metodología del estudio de clases, en la que se realizaron ejercicios de planeación, ejecución, observación y evaluación de las clases en equipos de estudios (comunidades de aprendizaje). De esta forma, los resultados obtenidos en esta experiencia educativa fueron la ayuda de los demás compañeros a mejorar la instrucción; en la experiencia Maestro, invítame a tu clase, de manera sencilla y tranquila, se observó y aprendió del otro; se trabajaron las clases demostrativas, en las que "un buen grupo de profesores ayuda a un compañero a preparar con anticipación un tema determinado, para luego trabajarlo con los estudiantes y allí evaluar y mejorar cada día" (Antioquia, 2007).

Continuando con las experiencias dentro de la implementación de la metodología estudio de clase y las comunidades de aprendizaje de docentes, se encuentra la experiencia investigativa Maestros que aprenden de
Evaluación de la propuesta

de enseñanza de las áreas de lenguaje y matemáticas en la

Institución Educativa San José del Pantano

I Panorama I pp. 25-39 I Volumen 9 I Número 17 I Julio-diciembre | 2015 
maestros, en Bogotá, desarrollada durante 2007, 2008 y 2009 en un nivel regional, que atendía al propósito de

desarrollar programas de formación docente, Alex Mauricio Díaz I a partir de las experiencias de aula de profesores de colegios públicos del Distrito Capital; algunos docentes que pertenecen al grupo de exbecarios de Japón, han socializado sus experiencias en el marco de la estrategia, lo cual ha permitido enriquecer las prácticas institucionales, no sólo de quienes participan en el Taller, sino de los maestros que los orientan, incentivando en otros maestros el deseo por conocer más sobre la estrategia "Estudio de Clase" (MEN, 2009, p. 73).

Los resultados obtenidos durante el desarrollo de esta experiencia investigativa estuvieron relacionados con el proyecto de implementación de actividades experimentales en las clases de ciencias naturales en la educación básica primaria y el cambio de actitud frente a las clases. Fue muy importante que los profesores terminasen el curso con una nueva disposición frente a las clases de ciencias naturales y matemáticas, ya que están dispuestos a explorar distintas formas de enseñar para motivar más y mejor a sus estudiantes. Y, por último, implementar nuevas estrategias didácticas en las clases de ciencias naturales y matemáticas en los primeros grados de educación formal, que son desarrolladas en comunidades de aprendizaje de docentes de todo el distrito capital.

Otra experiencia importante fue Estrategias pedagógicas y metodológicas para la cualificación docente, desarrollada por las docentes Carmen Ruby Rodríguez y Bertha Ligia Quintero de la Secretaría de Educación Municipal de Manizales (Caldas) en 2008, cuyo propósito era "implementar estrategias pedagógicas y metodológicas innovadoras que permitan mejorar las prácticas pedagógicas de los docentes de las instituciones Educativas del municipio de Manizales, beneficiarias de JICA (MEN,

Panorama I pp. 25-39।

Volumen 9 I

Número 17 | 2009 , p. 84). La metodología implementada durante esta experiencia educativa fue la focalización de siete instituciones educativa del municipio de Manizales del sector oficial, donde se dio el proceso de planeación, observación y evaluación por una comunidad de aprendizaje de 35 docentes de distintas áreas y niveles de educación. En este propósito, los resultados obtenidos y proyecciones fueron que los participantes entraran en contacto real con una metodología japonesa, pero adaptada a nuestro medio, fortaleciendo la capacitación maestro a maestro, la información y de la comunicación (TIC). Asimismo, se analizaron las ventajas de este sistema en el mejoramiento de los ambientes de aprendizaje en general. Además de institucionalizar las estrategias que plantea el proyecto en las instituciones vinculadas, articulándolas a los proyectos educativos institucionales (PEI) y dando continuidad a la formación de docentes en las estrategias propuestas en el proyecto investigativo.

La educación superior en Colombia también ha investigado sobre el impacto de la metodología del estudio de clase en los procesos de instrucción y de enseñanza por parte de los docentes en formación. Tal es el caso de la Universidad de Nariño, que, a finales de 2006, con la participación del docente adscrito al Departamento de Matemáticas, Gustavo Marmolejo ${ }^{1}$, orientó el proceso investigativo de la metodología de estudio de clase y comunidades de aprendizaje desde el nivel institucional de la educación superior, luego de asistir al proceso de formación situada en convenio con la JICA en Japón. El propósito de este proceso investigativo abarcaba la observación de clases planeadas y desarrolladas por estudiantes de octavo, noveno y décimo semestre del pregrado de Matemáticas a estudiantes de educación básica primaria en las instituciones educativas del sector oficial del departamento de Nariño, cuyos resultados obtenidos y las conclusiones derivadas por el investigador aportaron que la metodología de estudio de clase se constituyó en un referente a tener en cuenta en los programas de formación de profesores, ya que permite resaltar el papel que desempeña el trabajo grupal en el diseño de actividades de aula. Además, aporta experiencia en la implementación de actividades de aula en el desarrollo de planeaciones y en la realización de procesos de evaluación y autoevaluación de la práctica educativa, así como hace del aula de clase un lugar abierto a la crítica y posibilita su constante transformación.

La experiencia de implementación de la metodología de estudio de clase y las comunidades de aprendizaje que desarrollaron los docentes Richard Calvache ${ }^{2}$ y Fabián Escobar $^{3}$ en 2007 tuvo como objetivo analizar las clases desde los aspectos de la planeación, los referentes

\footnotetext{
1 Exbecario JICA 2006 y profesor del Área de Educación Matemática del Departamento de Matemáticas y Estadística de la Universidad de Nariño (Pasto, Colombia).

2 Docente de Matemáticas en educación básica de la Institución Educativa Las Mesas (El Tablón, Nariño).

3 Docente de Matemáticas en educación básica y el Programa de Formación Complementaria de la Escuela Normal Superior Enrique Vallejo (Páez Belalcázar, Cauca).
} 
didácticos y metodológicos empleados y su ejecución, para detectar problemas en la enseñanza de la temática y sugerir nuevas estrategias, y así mejorar su ejecución. Con un diseño metodológico de estudios de caso, reflexión y evaluación de la metodología de estudio de clase derivaron las siguientes conclusiones:

\begin{abstract}
No son pocos los beneficios aportados por el curso de mejoramiento ya que renacen las preocupaciones y la necesidad de cambiar esas viejas prácticas pedagógicas que aún redundan en muchas instituciones del país, ya sean de carácter oficial o privado y que van desde la zona urbana hasta la rural. El curso le abrió el espacio al maestro del sector oficial, para repensar su actuar docente, reflexionar en su historia como educador y vislumbrar nuevos horizontes, rutas y caminos. Aprender en conjunto con un grupo de maestros de la región sobre las formas de potenciar procesos de pensamiento en niños y jóvenes es de lo que se trata todo esto; estudiantes que vivencian sendas problemáticas en las aulas, con talentos desaprovechados, subestimados, subvalorados, con sueños truncados, a veces y en gran medida, por la baja preparación académica para acceder a programas de formación en Educación Superior. De ahí, que generar estrategias de capacitación internas en las instituciones, es una vía factible para mejorar la educación de la región paulatinamente (Calvache y Escobar, 2007, p.107).
\end{abstract}

Luego de todo esto proceso de experiencias educativas, y del convenio establecido entre el MEN con la JICA, se pasó a la socialización de todos los proyectos investigativos de la aplicación de la metodología de estudio de clase y las comunidades de aprendizaje de docentes y sus resultados en cada uno de los niveles ejecutados, dando lugar así al Primer Encuentro Nacional de Estudio de Clase en octubre de 2010, en el que se presentaron los avances logrados por los exbecarios de este curso, quienes participaron como expositores de sus experiencias, en las que habían aplicado las metodologías del estudio de clase, provenientes de Bogotá, Nariño, Caldas, Valle del Cauca, Antioquia, Cauca y Cundinamarca. Con este convenio internacional, la metodología del estudio de clases, la formación profesional docente y la atención de la educación básica primaria, se dio inicio a la implementación del programa Todos a Aprender ${ }^{4}$ en el país focalizando aspectos de pedagogía donde se privilegia la interacción comunicativa que se establece entre el docente y los estudiantes en contextos específicos.

En cuanto a la gestión desde un enfoque sistémico, esta facilita las relaciones entre los diferentes agentes y niveles, al tiempo que identifica y optimiza los recursos nacionales, regionales, institucionales y del entorno para imprimirle efectividad, sostenibilidad y eficacia a la estrategia. Por su parte, las condiciones básicas se refieren a los elementos necesarios para garantizar que el estudiante pueda estar inmerso en los ambientes de aprendizaje que le proponen las prácticas de aula, las cuales son transporte escolar, infraestructura física, alimentación escolar e infraestructura tecnológica. Por último, la formación situada se sustenta en el acompañamiento y en las problemáticas específicas del aula en torno a los procesos de aprendizaje de los estudiantes, enfocada en la estructuración de oportunidades para que el colectivo de maestros afine sus prácticas de aula; consecuentemente su desarrollo está relacionado con la creación, puesta en marcha y mejoramiento de ambientes de aprendizaje efectivos en contextos especialmente difíciles. Todo esto, con el objetivo de mejorar los niveles de desempeño de los estudiantes de las instituciones educativas del sector oficial ubicadas en zonas urbanas y rurales en las áreas de lenguaje y matemáticas en el nivel de la educación básica primaria.

Las investigaciones y experiencias reseñadas en este apartado demuestran la preocupación y el interés de todos los actores del sistema educativo. Del Gobierno y los establecimientos educativos de educación básica y superior por lograr el mejoramiento de la Instrucción Educativa, de la enseñanza y del aprendizaje de competencias necesarias para el mejoramiento de la calidad educativa en los ámbitos local, regional y nacional, de la educación inicial en las áreas de lenguaje y matemáticas. Por otro lado, se observa cómo los sistemas educativos que han implementado este tipo de metodología han logrado mejorar los niveles de enseñanza y aprendizaje en los maestros y los estudiantes, por lo que se han mejorado de forma inmediata las condiciones de vida en cada uno de estos países con altos niveles de desempeño académico en el mundo. De acuerdo con lo anterior, lo

\footnotetext{
4 Programa cuyo propósito es mejorar los aprendizajes de los estudiantes de básica primaria en lenguaje y matemáticas. Para lograrlo, acompañará 3000 establecimientos educativos y apoyará el quehacer formativo de 70000 docentes que trabajan con 2300000 estudiantes.
} 
Alex Mauricio

Díaz I

\section{Metodología}

La metodología de esta investigación es el estudio de casos que se centra en la conformación de un equipo de docentes, quienes se han planteado un plan de trabajo focalizado en un problema concreto de aula, como comprensión de contenidos, uso del material didáctico y las interacciones maestro-estudiantes en la clase, entre otros, con el fin de asegurar la pertinencia del proceso de enseñanza y la efectividad en la práctica frente al cumplimiento de los objetivos propuestos por el MEN y el programa Todos a Aprender. De esta forma, se lleva a cabo un estudio de la particularidad, singularidad y heterogeneidad de la práctica de los docentes desde tres fases: proyección de las comunidades de aprendizaje, ejecución del desarrollo profesional situado y consolidación de la comunidad de aprendizaje de docentes, en las que se aplica la reflexión y revisión de la práctica pedagógica constantemente (Valls, 2005).

La fase de proyección de la comunidad de aprendizaje de docentes comprendió el análisis y estudio de las metas propuestas por el programa Todos a Aprender para 2015, las cuales se centran en los aspectos de mejorar las condiciones de aprendizaje para los estudiantes matriculados entre transición y grado quinto de básica primaria, así como $25 \%$ de los estudiantes de la Institución Educativa debe ascender del nivel insuficiente, al menos en las áreas de lenguaje y matemáticas, en la prueba Saber $3^{\circ}$ y $5^{\circ}$ aplicación 2014. Esto fue acordado en el segundo semestre de 2013 mediante actas de sensibilización, acuerdos de gestión y socialización con toda la comunidad educativa de la Institución Educativa.

Volumen 91

Número 17 |

La fase de ejecución del desarrollo profesional situado de los docentes de la Institución Educativa consistió en las sesiones de trabajo situado que se desarrollan semanalmente con las comunidades de aprendizaje, en las que se planifican, ejecutan y evalúan las secuencias didácticas desarrolladas y observadas por todo el equipo de docentes de la básica primaria que conforman esta secuencias didácticas según el material educativo de lenguaje y matemáticas de acuerdo con el grado asignado para la sesión. Luego de este proceso, se procede a observar el desarrollo de la clase planeada por todo el equipo de trabajo, donde se aplica la metodología de estudio de clase con los instrumentos y protocolos diseñados para la observación de las clases, focalizando aspectos de instrucción, ambiente escolar, gestión del aula y conocimiento didáctico de los contenidos. Después de este proceso de observación, el mismo equipo de estudio de clase se reúne en comunidades de aprendizaje para socializar los resultados obtenidos, hacer correcciones, evaluar el proceso de instrucción y dar la retroalimentación final, para luego pasar al proceso inicial de planeación de secuencias didácticas en los problemas de aula observados en las reuniones anteriores. Todo este proceso constituye el ciclo de la metodología de estudio de clase (planeación, observación y evaluación de las clases en equipo), lo cual es la base pedagógica del programa Todos a Aprender.

Con el desarrollo profesional situado, también se pudo contribuir a un conocimiento más profundo del área disciplinar, pues, en los acompañamientos que se realizan en el aula de clase, el docente se apropia del contenido y, en consecuencia, aplica una didáctica específica para que el educando se apropie del conocimiento. Estas actividades permiten que los docentes exploren otras maneras de enseñar, así como maneras de construir el significado de los textos leídos. En cuanto al aprendizaje de los estudiantes, se comenzó a dar prioridad a lo que ellos deben aprender. La gestión académica se ha fortalecido gracias a que en las comunidades de aprendizajes se intercambian estrategias aplicadas y se buscan soluciones a problemas comunes e individuales relacionados con la práctica pedagógica.

La fase de consolidación de la comunidad de aprendizaje de docentes de la Institución Educativa se destaca por la importancia del desarrollo profesional situado y la metodología de estudio de clase para la atención de las necesidades educativas de los estudiantes y el mejoramiento de la calidad de la enseñanza de los docentes. De este modo, los docentes encuentran en las comunidades de aprendizaje la acción comunicativa y el aprendizaje dialógico, en los que se da un trabajo de enseñanza colaborativa y cooperativa. El desarrollo profesional situado ha servido en el programa Todos a Aprender como una fórmula para acercarse al análisis y discusión 
de las prácticas de aula. La estrategia es una iniciativa de gran importancia, porque con ella se ha dado un acercamiento entre los docentes para entablar diálogos pedagógicos y profesionales sobre la labor realizada. Por lo cual, es la constante evaluación del quehacer docente por pares académicos del mismo equipo de trabajo lo que permitirá la consolidación y acción de las comunidades de aprendizaje de docentes de la Institución Educativa San José del Pantano a lo largo del tiempo.

El desarrollo profesional situado ha tenido impacto dentro del trabajo del programa Todos a Aprender en la Institución Educativa, ya que se ha trabajado de manera conjunta los temas referentes a la planeación, ejecución, evaluación y reflexión de las prácticas docentes. Es en estas reflexiones, orientadas apropiadamente, donde ocurren algunos de los aprendizajes más significativos para los docentes, pues ellos mismos admiten que aciertos y desaciertos se han cometido durante su interacción académica con los estudiantes. También se puede considerar un avance la interacción entre los tutores y los docentes dentro del entorno del aula, lo cual permite observar en tiempo real lo que sucede en el aula, los aprendizajes obtenidos. Asimismo es importante destacar dentro de este esquema de desarrollo profesional la utilización del material pedagógico adecuado.

En el desarrollo de este estudio, se asume una perspectiva metodológica de tipo evaluativo, lo cual supone un "proceso de recogida de información orientado a la emisión de juicios de mérito o de valor respecto de algún sujeto, objeto o intervención" (Casarrubios et al., 2009,p. 2). Como marco orientador del estudio, la investigación permite un análisis del impacto del programa Todos a Aprender, en especial, de la comunidad de aprendizaje en la práctica de enseñanza del lenguaje y las matemáticas de los docentes de la Institución Educativa San José del Pantano.

El procedimiento metodológico seguido en este estudio es el siguiente:

1. 1) Revisión bibliográfica: en esta etapa se lleva a cabo un rastreo y revisión de las investigaciones asociadas a la metodología de estudio de clase y las comunidades de aprendizaje. Esta búsqueda sirve de marco orientador de la investigación sobre los objetivos, los alcances y la fundamentación teórica y metodológico del estudio.

2. 2) Aplicación de la encuesta: una vez terminado el rastreo bibliográfico, se procede a la aplicación de una encuesta a los docentes con el objeto de determinar la manera como ellos organizan la planeación de sus clases. Esto, por supuesto, constituye una primera etapa en el proceso investigativo, la cual es complementada con las etapas que a continuación se describirán.

A. Observación de las clases: el análisis de los cuestionarios realizados a los docentes permite inferir determinados aspectos de su clase. Esta información es complementada, precisada $\mathrm{y}$ revaluada con los datos obtenidos mediante la observación de la clase de los docentes. Dicha observación posibilita los estudios de caso de la práctica de los maestros.

B. Entrevistas a profundidad: a partir de los datos obtenidos con la encuesta y la observación de la clase de los docentes, se construye una entrevista. Esta entrevista es aplicada con el objeto de evaluar la manera como el docente desarrolla sus clases, los referentes educativos y materiales que utiliza y las experiencias significativas que construye con sus alumnos.

C. Análisis de los datos: como última etapa, se procede al análisis y triangulación de los datos obtenidos con la encuesta, la observación, la entrevista a profundidad y el resultado de las pruebas saber $3^{\circ}$ y $5^{\circ} 2014$.

\section{POBLACIÓN Y MUESTRA}

La población de esta investigación está compuesta por los dieciséis profesores de básica primaria de la Institución Educativa San José Del Pantano. La muestra está compuesta por siete docentes de básica primaria del establecimiento educativo. A causa del número reducido de maestros de la Institución Educativa en el nivel de básica primaria, se ha optado por elegir como muestra la totalidad de docentes de este nivel educativo, que constituyen la comunidad de aprendizaje con la cual se está trabajando.

Las técnicas de investigación utilizadas en el presente estudio son el cuestionario, la observación participante y la entrevista a profundidad. La observación participante es un elemento fundamental en los estudios de casos, es una técnica implícita en todas las técnicas utilizadas. La observación permite identificar el pensamiento del docente a través de la admiración de las acciones, por lo que se puede comprender mirando desde afuera lo que desde nuestro punto de vista parecía visible. Con la observación, se puede describir el quehacer docente en los procesos de enseñanza del lenguaje y las matemáticas, así como reconocer su realidad académica. Con este recurso metodológico, se va a recolectar información sobre las acciones que realizan los docentes
Evaluación de la propuesta

de enseñanza de las áreas de lenguaje y matemáticas en la

Institución Educativa San José del Pantano

I Panorama I pp. 25-39 I Volumen 9 I Número 17 I Julio-diciembre | 2015 
Alex Mauricio

Díaz I

cotidianamente, dentro o fuera de un aula de clases. Como lo afirma Castellanos (2012): "La operación del mirar implica otra, la de ad-mirar. Admiramos y, al adentrarnos en el admirado, lo miramos de dentro y desde adentro, lo que nos hace ver". Desde allí se parte hacia un proceso de comprensión y posterior re-significación del actuar en el proceso de enseñanza. Con esta técnica, se observan los hechos en forma no estructurada, se recogen impresiones generales de los sucesos, procesos, acontecimientos o situaciones naturales producidos espontáneamente en el entorno.

La entrevista a profundidad es una herramienta clave para conversar individualmente con los docentes acerca de criterios y valoraciones del accionar.

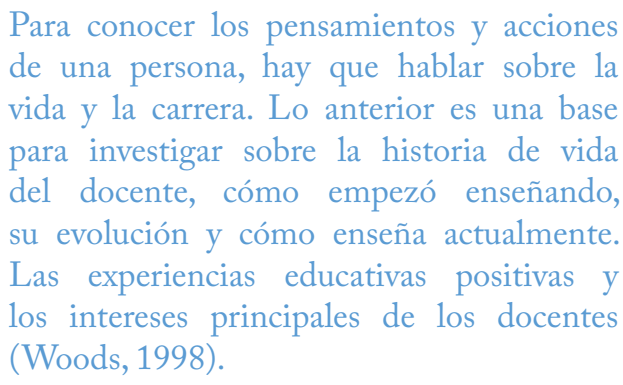

Por su parte, los instrumentos de investigación usados son el diario de campo, la grabadora y la cámara de video.

\section{RESULTADOS}

Dentro de esta investigación se realizó un análisis de tres aspectos que se focalizan en el programa Todos a Aprender, aplicado en la Institución Educativa San José del Pantano desde el segundo semestre de 2013, que son los siguientes: concepciones de los docentes integrantes de la comunidades de aprendizaje acerca del programa Todos a Aprender, el desarrollo profesional docente y el análisis comparativo de las pruebas Saber 2013-2014,

Panorama I pp. 25-39 I Volumen 9 I

Número 17 | Julio-diciembre | 2015 I calidad educativa y la instrucción docente en la institución educativa focalizada. parencia para la obtención de la información desde los diferentes actores, como docentes, directivos docentes y evaluadores externos. Además, una compilación de información, donde la intención es evaluar el impacto del programa en los aspectos de mejoramiento de la

\section{Concepciones de los docentes integrantes de la comu- nidades de aprendizaje acerca del programa Todos a Aprender}

La concepción está definida sobre las visiones y los conceptos que las personas tienen en torno a cualquier tema en particular de la vida. La concepción es un proceso mental que demanda un ejercicio cognitivo, hace referencia al vínculo que debe existir entre el conocimiento y las experiencias que fluyen en las prácticas de los maestros de lenguaje, en relación con el contexto donde realizan esas prácticas, lo que en términos de Rodríguez (2005, p. 76) hace referencia a que la "importancia recae en saber cómo los sujetos piensan o conceptúan los distintos fenómenos, cómo se concibe la tarea cognitiva que se tiene a mano". Atendiendo a que una concepción puede ser definida también como "la forma fundamental en la que la persona comprende los fenómenos del mundo que la rodean” (p. 77).

Según lo anterior, los docentes en sus intervenciones han demostrado una actitud receptiva desde la implementación del programa Todos a Aprender en la Institución Educativa, ya que ven en él una oportunidad de mejorar los procesos de enseñanza con la actualización pedagógica y didáctica mediante equipos de estudio de clase y de trabajo en comunidades de aprendizaje donde predomina el aprendizaje cooperativo y colaborativo a través de la acción comunicativa. Torres y Vergara (2009) expresan los objetivos que persigue el MEN en cuanto a los procesos de enseñanza de los docentes de educación básica primaria:

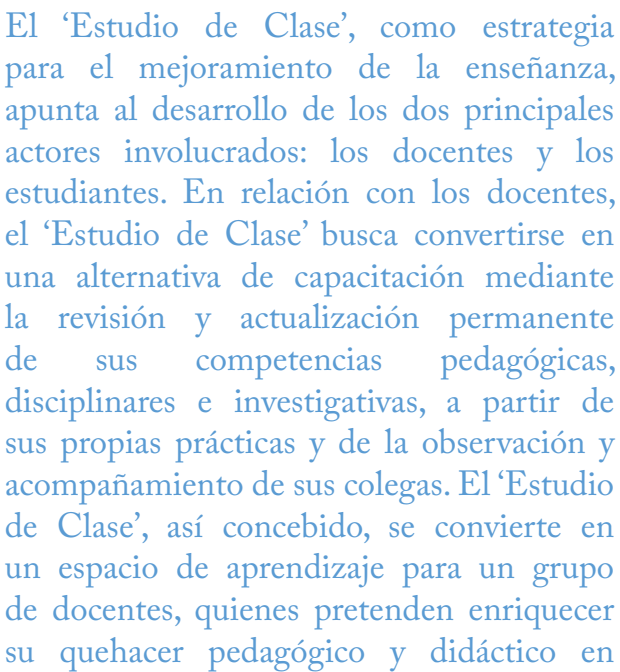


un ejercicio constante y colaborativo de análisis crítico del área o tema de estudio, planificación de las clases, observación de sus actuaciones, análisis de sus desempeños y retroalimentación conjunta. Así mismo, el 'Estudio de Clase' permite que los docentes compartan saberes y estrategias de trabajo con los estudiantes, frente a situaciones concretas de la realidad de las aulas (p. 31).

El planteamiento anterior demuestra que el sistema educativo actual está diseñado en torno a la formación docente en métodos, didácticas y conocimiento de los contenidos. Se busca reformular la escuela y sus procesos internos, repensar al maestro, entendiéndolo no como un actor pasivo del proceso educativo, sino como un indagador permanente de sus prácticas de enseñanza, en relación con los materiales y los referentes de la calidad educativa, como los lineamientos curriculares y los estándares básicos de competencias. Desde este texto del MEN, también se puede inferir que, para la búsqueda de la excelencia docente, ellos han de centrarse en tres elementos: la escuela como centro del conocimiento y cultura, el aula como espacio de investigación y de innovación constante y el maestro como sujeto autocrítico, investigador y trabajador en equipos de estudios y comunidades de aprendizaje.

En las entrevistas realizadas a los docentes acerca de la implementación del programa Todos a Aprender en la Institución Educativa, se ha encontrado que inicialmente este era percibido como una herramienta más del MEN, para saturar a las escuelas públicas del país y cumplir con las obligaciones de los organismos internacionales que rigen el rumbo educativo de este. Luego, a partir de la interacción continua con los docentes, se ha notado gran receptividad en cuanto a las metodologías que ha orientado el programa Todos a Aprender desde su aplicación en la Institución Educativa desde 2013, dado que es el contacto con el docente en el aula lo que genera un impacto significativo, y se ha encontrado que los docentes conciben el programa Todos a Aprender hoy día como un mecanismo útil para planear y evaluar sus clases de matemáticas y lenguaje con equipos de estudio de clases conformados por sus mismos compañeros de trabajo. Además de que deben cambiar su estrategia didáctica si sus prácticas no son efectivas (prueba de ineficacia), aprenden otras formas de enseñar y aprender (prueba de existencia), se dan cuenta de que pueden enseñar y hacerlo mejor cada día (prueba de capacidad). Y, por último, que el tiempo requerido para desarrollar y consolidar nuevas prácticas es de al menos entre dos a tres años (fase de consolidación de la comunidad de aprendizaje).

\section{DESARROLLO PROFESIONAL DOCENTE}

La formación pedagógica y actualización docente en el programa Todos a Aprender se centra en dos estrategias didácticas del Aprendizaje: aprendizaje colaborativo y aprendizaje cooperativo. El aprendizaje colaborativo desarrolla la participación, es decir, implica la conformación de pequeñas comunidades de aprendizaje. Las actividades colaborativas permiten ampliar el rango de interacción y trabajo en el aula y posibilitan acercarse a un problema o una situación haciendo conexiones cognitivas o sociales de manera diversa y diferenciada, lo cual enriquece los procesos cognitivos, afectivos, comunicativos y, por supuesto, la interacción social de los docentes que trabajan en equipo en la planeación, ejecución y observación de las clases. En este proyecto investigativo, se encontró que los docentes del área de humanidades fortalecieron sus debilidades en la didáctica de las matemáticas con el apoyo y sustento de los dos docentes licenciados en matemáticas, quienes durante las sesiones de trabajo situado en comunidades de aprendizaje retroalimentaron el proceso metodológico de las enseñanzas de las matemáticas basados en los procesos y pensamientos matemáticos, así como los docentes en humanidades fortalecieron el quehacer pedagógico en las prácticas educativas de lenguaje.

De esta manera, para la formación docente, los resultados que se han obtenido desde el aprendizaje colaborativo están relacionados con los procesos de enseñanza, es decir, desde el centro de las comunidades de aprendizaje de docentes las fortalezas que tienen algunos profesores por su experiencia, conocimiento pedagógico y disciplinar les ha permitido al resto de los docentes aprender y dar solución a los problemas que se presentan en la enseñanza dentro de las aulas a partir de las sesiones de trabajo situado, el equipo de estudio y la metodología de estudio de clase, donde se han identificado las debilidades en la enseñanza, aspectos que luego son discutidos y trabajados entre todos los docentes, para luego pasar al aula de clases y observar cómo ha funcionado la estrategia didáctica, a fin de dar solución a la situación en cuestión. De este modo, se genera aprendizaje significativo, colaborativo y cooperativo en el equipo de trabajo de docentes. En las prácticas colaborativas, los docentes se
Evaluación de la propuesta

de enseñanza de las áreas de lenguaje y matemáticas en la Institución Educativa San José del Pantano

I Panorama I pp. 25-39 I Volumen 9 I Número 17 I Julio-diciembre | 2015 
Alex Mauricio

Díaz I benefician, porque disminuyen su aislamiento e incertidumbre y conforman verdaderas comunidades de apoyo, más productivas y críticas sobre las prácticas, hacia una verdadera gestión del cambio, con la actualización pedagógica que se trabaja en los equipos de estudio y las sesiones de trabajo situado, donde se observan las clases y se debate en torno a una problemática previamente establecida. Es decir, gestión del tiempo del aula, ambiente escolar, conocimiento didáctico de los contenidos y estrategias pedagógicas eficaces y efectivas para la instrucción docente, que demuestran un proceso significativo de la instrucción y del quehacer docente en nuestras aulas de clases de la Institución Educativa San José del Pantano. En segundo lugar, se pueden rescatar como resultados de la formación docente el liderazgo, el sentido de responsabilidad que se despierta por el aprendizaje propio y el de los demás, la tarea como producto de una acción conjunta del grupo, el análisis e identificación de las formas como se aprende y aprenden los demás y la compañía del docente como observador de las dinámicas, mediador en el aprendizaje y orientador de las acciones promovidas para la enseñanza a partir de las experiencias adquiridas por el papel docente o la formación in situ que se trabaja durante las reuniones semanales dentro de las orientaciones del programa Todos a Aprender, donde se desarrollan aspectos de pedagógica, didáctica y evaluación mediante simulaciones de clases con juegos de roles de los docentes participantes.

Por último, estas prácticas promueven el desarrollo profesional y la afirmación de la identidad del maestro y su lugar como miembro valioso de la comunidad educativa. El trabajo colaborativo y cooperativo de los profesores en los procesos de planeación, enseñanza y evaluación promueven mejores prácticas pedagógicas, mejoran el clima institucional y promueven la creatividad y el desarrollo profesional docente. Además, el poder de la observación como acompañamiento a clase para explo-

Panorama I pp. 25-39 I Volumen 9 । Número 17 I rar el estudio de clase y la práctica y sus protagonistas como objeto de reflexión e investigación.
ANÁLISIS COMPARATIVO DE LAS PRUEBAS EXTERNAS 2013-2014

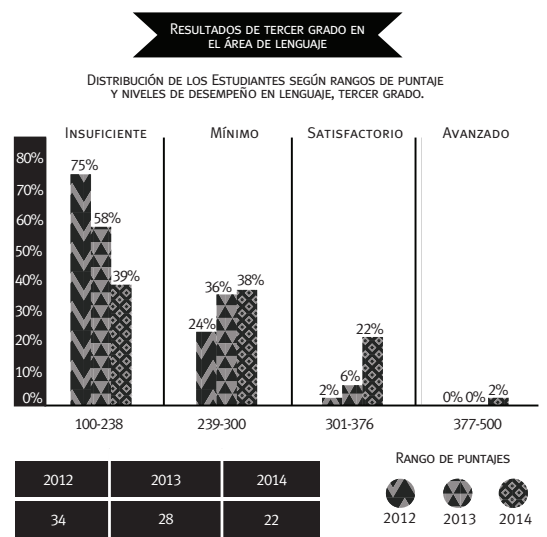

Figura 1. Resultados de tercer grado en el área de lenguaje

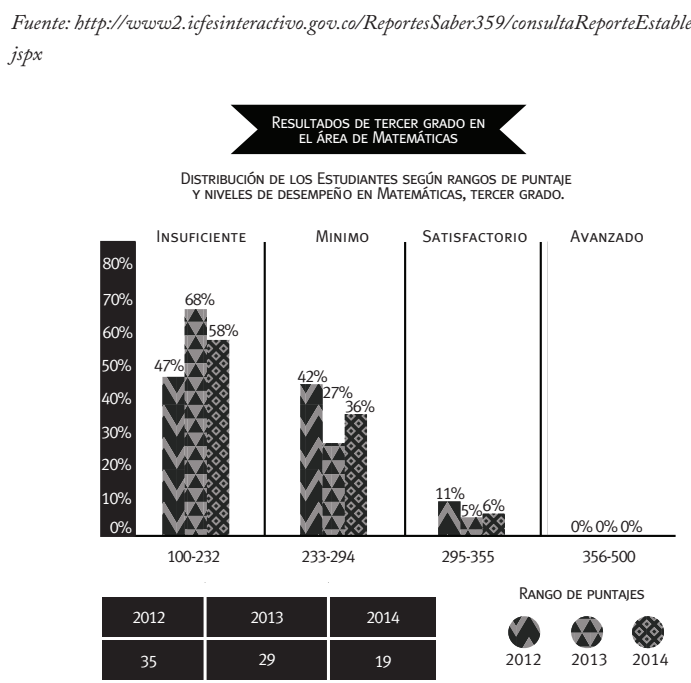

Figura 2. Resultados de tercer grado en el área de matemáticas

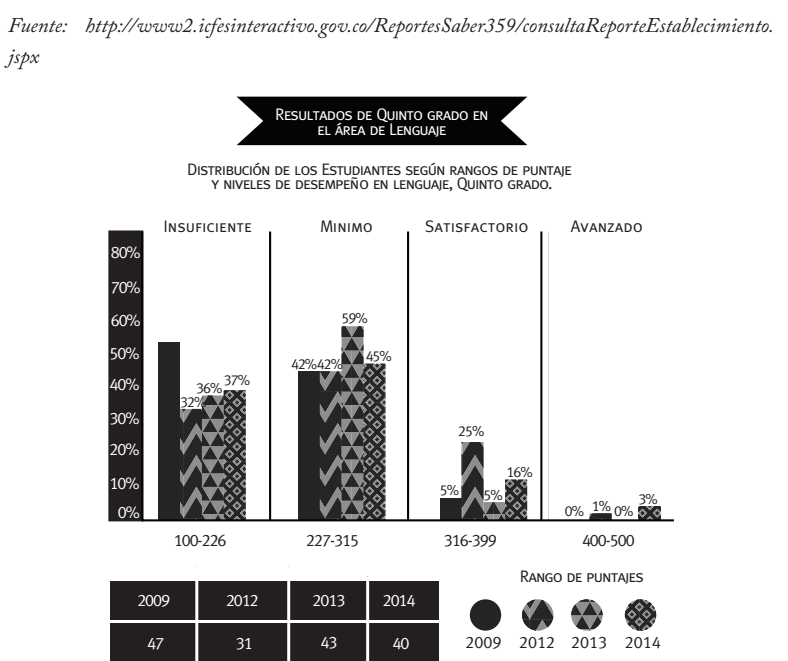

Figura 3. Resultados de quinto grado en el área de lenguaje Fuente: http://www2.icfesinteractivo.gov.co/ReportesSaber359/consultaReporteEstablecimiento. $j s p x$ 


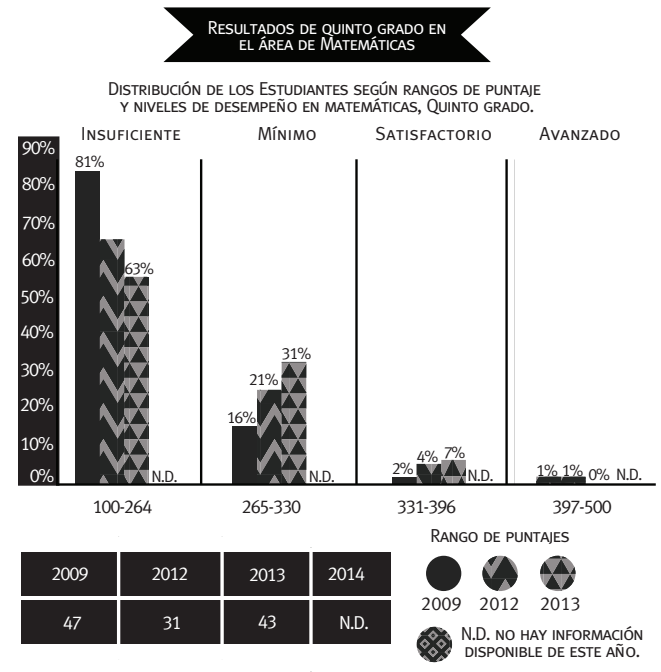

Figura 4. Resultados de quinto grado en el área de matemáticas

Fuente: http://www2.icfesinteractivo.gov.co/ReportesSaber359/consultaReporteEstablecimiento. $j s p x$

Al comparar los resultados de la prueba externa Saber $3^{\circ}$ y 5o ciclo 2012-2014 e históricos 2009 de la misma prueba en el área de lenguaje, se evidencia un comportamiento ideal para las aspiraciones institucionales de la Institución Educativa, donde se puede decir que, en primer lugar, se cumplieron las metas planteadas por el programa Todos a Aprender de mejorar las condiciones de aprendizaje para los estudiantes matriculados entre transición y quinto grado de básica primaria. En segundo lugar, $25 \%$ de los estudiantes de la Institución Educativa San José del Pantano debían ascender del nivel insuficiente en los resultados de las pruebas Saber 2014, lo cual se alcanzó satisfactoriamente. También se espera que para las pruebas Saber 2015 se haya reducido totalmente el desempeño insuficiente en la Institución Educativa; en otras palabras, en grado tercero y quinto se espera que todos los estudiantes superen las preguntas de menor complejidad de la prueba.

En el área de matemáticas, este comportamiento también se evidencia mediante un descenso porcentual considerable en el desempeño insuficiente entre los resultados de la prueba Saber 2013 (68 \%) y la prueba Saber 2014 (36\%). Lo anterior se puede interpretar como una mejora significativa en el área de matemáticas en grado tercero si se tiene en cuenta que reduce el porcentaje de estudiantes con desempeño insuficiente, aumenta el porcentaje de estudiantes con desempeño mínimo y ubica $6 \%$ del total de estudiantes en desempeño satisfactorio, resultado que es coherente con las metas que persigue el programa Todos a Aprender en las instituciones educativas focalizadas en todo el país. Al comparar la prueba interna con el histórico de la prueba externa, se evidencia una disminución de $26 \%$ en el desempeño insuficiente, un aumento de $27 \%$ en el desempeño mínimo y se mantiene estable el desempeño satisfactorio. Lo que evidencia el mejoramiento de los procesos de instrucción y de enseñanza en los docentes en las áreas evaluadas y la consecución de las metas establecidas por el programa para el establecimiento educativo en los acuerdos de gestión firmado por los docentes y directivos docentes en su iniciación desde 2013.

\section{CONCLUSIONES}

Para propiciar una comprensión de las generalidades del proceso investigativo y de las particularidades del contexto en el cual se encuentra inmersa la institución educativa San José del Pantano y satisfacer la problemática que ha iluminado este proyecto investigativo, se focalizan los aspectos fundamentales de la percepción de los docentes hacia el programa Todos a Aprender, la formación docente y el análisis comparativo de los resultados de las evaluaciones internas y externas a partir de la implementación de su implementación desde el segundo semestre de 2013 en la Institución Educativa. Desde lo cual se desprenden las siguientes conclusiones.

En primer lugar, se comprende que las concepciones de los docentes de la Institución Educativa San José del Pantano ante la implementación del programa Todos a Aprender inicialmente fueron las siguientes: capacitación aislada y descontextualizada, programa del MEN para la persecución del docente y su quehacer pedagógico, información recolectada a través de la entrevista y la pregunta abierta. Sin embargo, con el paso del tiempo han encontrado en el programa la herramienta para el desarrollo profesional situado y equipos de estudios desde la comunidades de aprendizaje para debatir problemas que se presentan en el aula durante el proceso de enseñanza. Además de la planeación, ejecución, observación y evaluación-reflexión de las clases por un equipo de expertos internos del establecimiento educativo o de pares académicos, quienes mediante la formación docente, el aprendizaje autónomo, colaborativo y cooperativo se han constituido en agentes expertos para emitir juicios y reflexionar sobre las sesiones de clases desarrolladas y evaluadas por cada uno de los docentes
Evaluación de la propuesta de enseñanza de las áreas de lenguaje y matemáticas en la Institución Educativa San José del Pantano
I Panorama I pp. 25-39 I Volumen 9 I Número 17 I Julio-diciembre | 2015 
de las comunidades de aprendizaje de la Institución Educativa San José del Pantano.

Alex Mauricio

Díaz I

En segundo lugar, la reflexión constante sobre los procesos de enseñanza del área de lenguaje en la educación básica primaria, utilizados por los docentes de las comunidades de aprendizaje, es una forma significativa de capacitación, aprendizaje significativo y actualización pedagógica que, a diferencia de los cursos de capacitación aislados, compromete la participación conjunta de los miembros de la comunidad educativa a partir de la implementación de las sesiones de trabajo situado y de desarrollo profesional orientado bajos las políticas de prácticas educativas y de calidad del programa Todos a Aprender.

Por tal razón, Doria y Pérez (2008) exponen:

$$
\begin{aligned}
& \text { Asumir la importancia del diálogo } \\
& \text { intelectual y profesional entre pares, que } \\
& \text { son capaces de reconocerse y reconocer sus } \\
& \text { aportes y experiencias académicas, en medio } \\
& \text { de las diferencias ideológicas, tal como } \\
& \text { sucedió en los talleres del GET (Grupo de } \\
& \text { Estudio Trabajo) asumidos como espacios } \\
& \text { para colectivizar el debate sobre el tema de } \\
& \text { lectura y escritura. }
\end{aligned}
$$

Finalmente, a partir del análisis comparativo entre las pruebas externas Saber 2013 y 2014 (referencias saber $3^{\circ}$ y saber $5^{\circ}$ ), podemos concluir que en relación con las áreas del lenguaje y las matemáticas la Institución Educativa San José del Pantano de Puerto Escondido (Córdoba) ha llevado a cabo acciones concretas por parte de los directivos y docentes orientadas hacia el mejoramiento de la calidad educativa de esta y, en especial, de las prácticas de enseñanza y evaluativas de los docentes. Lo cual se traduce en un compromiso constante de los profesores por generar espacios de reflexión, capación y retroalimentación sobre las estrategias de enseñanza y

Panorama I pp. 25-39। Volumen 9 I Número 17 | muestran que los docentes han implementado de manera rigurosa y regulada estrategias y criterios evaluativos acordes con las nuevas políticas, lineamientos y orientaciones pedagógicas establecidos por el MEN dentro del programa Todos a Aprender en relación con las prácticas evaluativas, los cuales son necesarios para que las escuelas alcancen los índices de calidad educativa que propone el MEN. estándares, ejes y procesos de pensamiento que articulan las áreas de lenguaje y matemáticas. Estos resultados indican que el trabajo sobre y desde la metodología del estudio de clases (metodología de estudio de clase) en la institución obedece a unos lineamientos claros de los directivos y docentes preocupados por el futuro de los niños, quienes, y a diferencia de los de años anteriores, cuentan con mejores y mayores herramientas para afrontar las pruebas Saber-Icfes de grado $3^{\circ}$ y $5^{\circ}$, dado que se hacen trabajos de preparación y alistamiento a través de simulacros, evaluaciones diagnósticas y evaluación por competencias con los estudiantes desde el trabajo en equipo de la comunidad de aprendizaje de docentes de la Institución Educativa.

Podemos concluir, entonces, que en la Institución Educativa San José del Pantano de Puerto Escondido (Córdoba) las actividades y orientaciones realizadas desde el marco legal y operativo del programa Todos a Aprender del MEN, a través de la comunidad de aprendizaje de docentes, la metodología del estudio de clases y la evaluación formativa han tenido su efecto e impacto positivo sobre la enseñanza e instrucción orientada por los docentes de básica primaria, en especial, en relación con sus metodologías, estrategias didácticas, desarrollo profesional y prácticas evaluativas.

\section{REFERENCIAS BIBLIOGRÁFICAS}

1. Agudelo, J. D. (2007). Estudio de áreas y perimetro dentro de una cuadricula haciendo uso de la tecnología. Manuscrito no publicado.

2. Alcaldía Mayor de Bogotá, Secretaría de Educación de Bogotá (2007). Colegios públicos de excelencia para Bogotá. Recuperado de http://www.educacionbogota.edu.co/ Centro_Documentacion/anexos/publicaciones_2004_2008/101084-Pensamiento \%20 Historico \%20Feb \%201.pdf.

3. Calvache y Escobar. (2007). Experiencia No. 5: 'Estudio de clase - Transformaciones Rígidas' Cauca - Año 2007. Nivel Regional. Convenio MEN Colombia-Agencia JICA

4. Casarrubios, M. ${ }^{\text {a T., García Simón, B., Mejías }}$ Gómez, A., San Cristóbal, N. y Senín Velasco, V. (2009). Métodos de investigación en educación especial. Recuperado de http://uam.es/ personal_pdi/stmaria/jmurillo/InvestigacionEE/ Presentaciones/Inv_evaluativa_ppt.pdf 
5. Castellanos, C. (2012). Los pensamientos de los profesores universitarios: las concepciones pedagógicas sobre la enseñanza y el aprendizaje en la Facultad de Ciencias de la Salud (Tesis de maestría, Universidad de Córdoba, Montería, Colombia).

6. Chile (2011). Campaña de lectura, escritura y matemática. Estrategia LEM. Recuperado de http://www.preal.org/BibliotecaDes. asp? id=2187\&Camino=319| $\mid$ Programas $/ 321 \mid-$ BuenasPracticas/104|Buenas\%20Practicas\%20 Politica\%20Educacional\%20y\%20Reforma\%20 Educativa\%20en\%20America\%20Latina/106|Experiencias\%20registradas\%20 seg\%FAn\%20pa\%EDs/114|Cono\%20Sur

7. Doria R.y Pérez T. (2008). Prácticas de enseñanza de lectura y escritura en instituciones educativas de la ciudad de Montería: concepciones y metodologias (Tesis de maestría, Universidad de Córdoba, Montería, Colombia).

8. EXPERIENCIA No. 1: 'Maestro Invítame a tu Clase'. El grupo de 'Estudio de Clase' de la Institución Educativa E1 Hatillo y el desarrollo de clase en la Básica Primaria. BarbosaAntioquia. Año 2007 - 2008. Nivel Institucional. Convenio MEN Colombia - Agencia JICA.

9. Freire, P. (1992/1993). Pedagogía de la esperanza. México: Siglo XXI.

10. Habermas, J. (1984/1993). Teoría de la acción comunicativa: complementos y estudios previos. Madrid: Cátedra.

11. Hu-pei \& Fullan, (2005). Understanding teacher development. New York: Teachers College Press.

12. McKinsey (2007). How the World's BestPerforming School Systems Come out on Top. Recuperado de http://www.smhccpre.org/wp-content/uploads/2008/07/ how-the-worlds-best-performing-school-systems-come-out-on-top-sept-072.pdf

13. Ministerio de Educación Nacional (2006). Estándares básicos de competencias en lenguaje, matemáticas, ciencias y ciudadanas. Bogotá. Recuperado de http://www.mineducacion.gov. co/1621/articles-116042_archivo_pdf.pdf

14. Ministerio de Educación Nacional (2009a). Curso Metodología Estudio de Clase. Recuperado de https://www. youtube.com/watch?v=_NQaB 4 aDGlo

15. Ministerio de Educación Nacional (2009b). Mejoramiento del sistema de enseñanza de docentes de matemáticas y ciencias naturales. Bogotá. Recuperado de https://www.academia. edu/7832835/229447158-Libro-Estudio-de-Clase-Colombia-Men
16. Ministerio de Educación Nacional (2011).

Programa para la Transformación de la Calidad

Educativa. Guía para actores involucrados en el Programa. Recuperado de http://www. mineducacion.gov.co/1621/articles-266934 archivo_pdf_documento_transformacion.p $\overline{d f}$

17. Ministerio de Educación Nacional (2012). Propuesta para la consolidación de los módulos en didáctica del lenguaje y las matemáticas en el marco del componente de formación situada.

18. Mitchell, C., \& Coral. (2001). Profound improvement: Building capacity for a learning community. Lisse, NL: Swets \& Zeitlinger.

Evaluación de
la propuesta
de enseñanza
de las áreas
de lenguaje y
matemáticas
en la
Institución
Educativa
San José del
Pantano

19. Nelson, T. y Slavit, D. (2008). Supported Teacher Collaborative Inquiry. Recuperado de http://www.teqjournal.org/Back \%20 Issues/Volume \%2035/VOL35 \%20 PDFS/35_1/14nelson\&slavit-35_1.pdf

20. Nunan, D. (2003). Collaborative Language Learning and Teaching. Cambridge: Cambridge University Press.

21. Roberts, Pruitt, Du Four y Eaker, (1998). Collaborative Activities and Strategies for Professional Development. high standards set by the profession; National Staff Development Council.

22. Rodríguez Rodríguez, Lourdes (2005). Análisis de las creencias epistemológicas, concepciones y enfoques de aprendizaje de los futuros profesores (Tesis doctoral). Departamento de Psicología Evolutiva, Facultad de Educación, Universidad de Granada, Granada, España.

23. Sistema de Capacitación docente en Japón (s. f.). Maestros aprendiendo juntos. Recuperado de https://www.youtube.com/ watch?v=kBP81pQqtY8

24. Torres, J. A. y Vergara Beltrán, L. I. (2009). Estudio de clase: una experiencia en Colombia para el mejoramiento de las prácticas educativas. Bogotá: MEN.

25. Valls, R. (2005). Educación social en la escuela. Infancia y Educación Social, 4. Recuperado de http://www.eduso.net/res/?b=7\&c=53\&n=145

26. Vezub, L. F. (11 de Noviembe de 2009). Organización de las Naciones Unidas para la Educación, la Ciencia y la Cultura (Unesco). Obtenido de Instituto Internacional de Planeamiento de la Educación (IIPE): http:// www.iipe-buenosaires.org.ar

I Panorama

I pp. 25-39

27. Wellman \& Calhoun (2005). Teacher Professional Learning And Pre-Service Teacher Education: Building A Sustainable Community. Journal of Teacher Education and Training, 5, 3-15.

I Volumen 9

I Número 17

28. Woods, P. (1998). Investigar el arte de la enseñanza: el uso de la etnografía en la educación. Barcelona: Paidós Ibérica. 